\title{
FINAL REPORT OF DOE GRANT NO. DE-FG03-94ER54271
}

(I) Principal Investigator: Liu Chen

(II) Title: "Theoretical Studies of Drift-Alfvén and Energetic-Particle Physics in Fusion Plasmas"

Period: 06/01/2002-11/30/2004

(III) Research Activity and Journal Publications

(1) "Electron temperature gradient instability in toroidal plasmas" -

Electron temperature gradient (ETG) driven instability in toroidal plasmas is studied with gyrokinetic theory. The full electron kinetics including finite Larmor radius effects, toroidal (curvature and magnetic gradient) drift motion $\omega_{D}$, and transit $k_{\|} v_{\|}$, is considered. The upgraded numerical scheme for solving the integral eigenvalue equations allows the study of both growing and damping modes, and thus direct calculation of critical gradient. A systematic parameter study is carried out for low $\beta$ (=plasma pressure/magnetic pressure) circular flux surface equilibria. The basic characteristics of the modes are discussed. The scaling of the critical gradient with respect to toroidicity and to the ratio of electron temperature over ion temperature is obtained. Estimation for the transport induced by the modes is given.

Publication: Physics of Plasmas 9, 4699 (2002).

(2) "Energetic particle mode stability in tokamaks with hollow q-profiles" -

A thorough analysis of energetic particle modes (EPM) stability and mode structures is presented for tokamaks with hollow $q$ profiles. Focusing on the region near the minimum- $q$ surface, EPM gap modes and resonant EPMs are shown to exist as solutions of the same dispersion relation. By controlling the fast ion distribution function, or, equivalently, their fundamental dynamical properties, a smooth transition between these two classes of modes is obtained within the EPM dispersion relation. When toroidal coupling becomes important, it is demonstrated that EPMs may have either single or double hump radial structures. The local analyses of EPM stability and mode structures near the minimum- $q$ surface are put in the broader framework of EPM stability and EPM induced transport in tokamaks with hollow $q$ profiles and a brief summary is also given of present understanding of such problems based on results of three-dimensional nonlinear hybrid magnetohydrodynamic-gyrokinetic simulations. Possible implications of present results 
are discussed in terms of experimental observations and possibilities of designing novel experimental setups to probe, at least conceptually, the complex predictions of theory. Publication: Physics of Plasmas 9, 4939 (2002).

(3) “Nonlinear saturation of high-m Alfvén-ballooning modes in magnetospheric plasmas”-

A theoretical model is proposed for the nonlinear saturation of high-m Alfvén-ballooning instabilities in in magnetospheric plasmas. Here, $\mathrm{m}$ is the azimuthal wave number. In the present model, a broad spectrum of Alfvén waves nonlinearly generate ion-sound density perturbations; which, in turn, scatter the Alfvén turbulence toward lower frequencies. Balancing the linear instability growth rate with the nonlinear scattering (Landau damping) rate then yield the corresponding saturated spectrum, which tends to peak near the bottom of the eigenmode frequencies. The theory also gives estimates of wave amplitudes at saturation in reasonable agreement with satellite observations.

Publication: Geophysical Research Letters 30, doi:10.1029/2002GL016065 (2003).

(4) "Kinetic theory of geomagnetic pulsations: 4. Hybrid gyrokinetic simulation of drift-bounce resonant excitation of shear Alfvén waves”-

A one-dimensional linear hybrid gyrokinetic-magnetohydrodynamic $\delta f$ particle in cell simulation code is developed to study the detailed mechanisms of energetic particle drift-bounce resonant destabilization of Alfvén modes in the ring-current region of the magnetosphere. Full kinetic effects such as finite Larmor radii and particle magnetic bounce and precessional drift motions are retained nonperturbatively. A simple finite $\beta$ dipolar equilibrium model is assumed ( $\beta$ is the ratio between plasma and magnetic pressures). Simulations show excellent agreement with earlier perturbative analyses. Results show that when the energetic ion thermal velocity is super-Alfvénic, the ions destabilize both odd and even parity shear Alfvén MHD modes via the drift-bounce resonances. The growth rates of the resulting modes scale linearly with plasma $\beta$. The most unstable of these modes are found to be drift-bounce resonance destabilized modes with odd parity, with wave numbers such that $\kappa_{\perp} \rho \approx 0.5$ at the equator ( $\rho$ is the energetic ion Larmor radius). The destabilization typically occurs at a critical wave number $\kappa_{\perp} \rho \approx 0.3$. When the wave number is close to this critical value and the plasma $\beta$ is close to the ideal MHD critical value, the mode frequency is determined by the energetic particle dynamics similar to the energetic particle modes (EPMs) observed in laboratory fusion plasma experiments. 
Publication: Journal of Geophysical Research 108, doi:10.1029/2002JA009650 (2003).

(5) "Zonal-Flow Dynamics and Size Scaling of Anomalous Transport”-

Nonlinear equations for the slow space-time evolution of the radial drift-wave envelope and zonal flow amplitude have been self-consistently derived for a model nonuniform tokamak equilibrium within the coherent four-wave drift wave-zonal flow modulation interaction model of Chen, Lin, and White [Phys. Plasmas 7, 3129 (2000)]. Solutions clearly demonstrate turbulence spreading due to nonlinearly enhanced dispersiveness and, consequently, the devicesize dependence of the saturated wave intensities and transport coefficients.

Publication: Physical Review Letters 92, 075004 (2004).

(6) "Nonlinear Paradigm of Drift Wave-Zonal Flow Interplay: Coherence, Chaos, and Turbulence" -

Starting from the coherent four-wave drift wave-zonal flow modulation interaction model of Chen, Lin, and White [Phys. Plasmas 7, 3129 (2000)], nonlinear equations for drift wave-zonal flow interplay are systematically derived from first principles for nonuniform toroidal plasmas. The paradigm model, presented here, is based on a hierarchy in nonlinear wave-wave couplings, which assumes that different drift wave interactions on the shortest nonlinear time scale are mediated by zonal flows. The resulting coherent model demonstrates turbulence spreading to be the cause of transport scaling with system size. The nonlinear saturated state can be either coherent, with limit cycles, or chaotic, depending, depending on the proximity to marginal stability.

Publication: Physics of Plasmas 11, 2488 (2004).

(7) "Finite Gyroradius Theory of Drift-Compressional Modes" -

A dispersion relation for ultra-low frequency drift compressional modes in high-pressure plasmas with finite gyroradius is derived from the linearized gyrokinetic -Maxwell equations with cold electrons and narrow eigenmode localization width along the field line. The dispersion relation demonstrates instability under two different conditions: 1) when the density gradient and proton temperature gradient are in opposite directions, or (2) when the magnetic guiding center drift is reversed with respect to the proton diamagnetic drift, i.e., drift reversal, which could occur during periods of strong magnetospheric disturbance. Furthermore, it is found that the most unstable modes have short azimuthal wavelengths comparable to the proton gyroradius. Publication: Geophysical Research Letters 31, L17804 (2004). 
